\title{
Hypertension as the trigger for posterior reversible encephalopathy syndrome in paediatric renal patients: An important diagnosis that should not be missed
}

\author{
J K Strong, MB ChB; K L Petersen, MB ChB, FC Paed (SA), DCH (SA), Cert Nephrology (SA); U Kala, MB ChB, FC Paed (SA), DCH (SA) \\ Department of Paediatrics, Chris Hani Baragwanath Academic Hospital and Faculty of Health Sciences, University of the Witwatersrand, \\ Johannesburg, South Africa
}

Corresponding author: J K Strong (jessie.rule@gmail.com)

\begin{abstract}
Background. Posterior reversible encephalopathy syndrome (PRES) is a reversible neurological condition presenting with seizures and visual disturbances and diagnosed on magnetic resonance imaging (MRI). Little is understood about its pathogenesis, particularly in children, but it is thought to be related to hypertension.

Objectives. To review the presentation, diagnosis and outcome of PRES in paediatric renal patients at Chris Hani Baragwanath Academic Hospital, Johannesburg, South Africa, between 1 January 2000 and 31 January 2017 and compare these with published case reports to date. Methods. This was a retrospective analysis of five new cases and a review of the existing literature.

Results. The five reported patients were all hypertensive at the time of diagnosis and presented with seizures. Most (91\%) of the 64 reviewed patients were also hypertensive at initial presentation. All five of the reported and $91 \%$ of the reviewed patients presented with seizures. The most common pattern of change on MRI occurred in the parietal and occipital regions. Complete neurological recovery occurred in four of the five reported and $87.5 \%$ of the reviewed patients.

Conclusion. All patients presented clinically with hypertensive crises and radiological evidence of PRES. Seizures were the most common presenting symptom. The prognosis for paediatric patients with PRES is favourable, so it is important to confirm the diagnosis in lowresource settings where intensive care is limited.
\end{abstract}

S Afr Med J 2018;108(4):325-328. DOI:10.7196/SAMJ.2018.v108i4.12816

Posterior reversible encephalopathy syndrome (PRES) is a neurological condition first described in $1996^{[1]}$ and defined as reversible subcortical vasogenic brain oedema presenting with neurological symptoms ${ }^{[2]}$ including encephalopathy, seizures, headaches and visual disturbances. The diagnosis is confirmed radiologically by decreased diffusivity of cortical and subcortical brain tissue of T2-weighted magnetic resonance imaging (MRI) ${ }^{[2,3]}$ The two main hypotheses of the pathogenesis of PRES are related to factors influencing cerebral perfusion and endothelial dysfunction. ${ }^{[4]}$

Despite a growing number of studies describing PRES, the risk factors, symptomatology and pathophysiology are not well understood. Although PRES is well documented in the adult population, little is known about the disease process in children. A limited number of PRES diagnoses associated with renal disease in paediatric patients have been described in the literature to date, and all of these are case reports. ${ }^{[5-17]}$

The diagnosis of PRES is important because the prognosis is generally favourable ${ }^{[18]}$ and these children should be offered intensive care even in low-resource settings, where misdiagnosis may mean that they would be considered poor candidates. The diagnosis is usually made on MRI when predisposing factors and symptoms are suggestive, but it may be more sensitively detected by an electroencephalogram showing focal non-convulsive seizures or status epilepticus. ${ }^{[18]}$

We postulate that as a disease entity PRES is not entirely separate from hypertensive encephalopathy and may represent a radiographically imaged and more severe presentation of the same disease process, now identifiable because of the availability of MRI. We therefore reviewed the presentation, diagnosis and outcome of PRES in paediatric renal patients at Chris Hani Baragwanath Academic Hospital, Johannesburg, South Africa, and compared these with published case reports.

\section{Methods}

We retrospectively report on the records of five children with renal disease diagnosed with PRES at Chris Hani Baragwanath Academic Hospital between 1 January 2000 and 31 January 2017. Data pertaining to their demographics, underlying diagnosis, medical therapy, biochemical and haematological parameters, blood pressure, clinical presentation, radiological findings and outcome are detailed. This information is compared with all published articles on PRES in paediatric renal patients in the PubMed database. The search terms 'posterior reversible (leuko)encephalopathy syndrome' AND 'paediatric', 'pediatric' OR 'child' AND 'renal' OR 'kidney' were used. Ethical permission was obtained from the Human Research Ethics Committee, University of the Witwatersrand, Johannesburg (ref. no. M1611153).

\section{Setting}

Chris Hani Baragwanath Academic Hospital is a tertiary institution in Soweto, Johannesburg, affiliated with the University of the Witwatersrand. The paediatric renal department sees $\sim 1500$ patients per year, and admits $\sim 30$ patients per month.

\section{Results}

Case 1

An 11-year-old girl on peritoneal dialysis (PD) for chronic kidney disease $(\mathrm{CKD})$ due to granulomatosis with polyangiitis developed 
generalised tonic-clonic seizures, right-sided hemiplegia and cortical blindness. These symptoms deteriorated over 2 days, and she became non-responsive and required ventilatory support. Prior to onset of the above symptoms there had been worsening control of her blood pressure, which was $160 / 110 \mathrm{mmHg}$ at its highest (50th centile 101/60 mmHg), ${ }^{[19]}$ and worsening biochemical markers of renal function while on PD (blood urea level $10.6 \mathrm{mmol} / \mathrm{L}$, serum creatinine $844 \mu \mathrm{mol} / \mathrm{L}$, serum sodium $149 \mathrm{mmol} / \mathrm{L}$ ). She had completed a course of empirical antibiotics for suspected bacterial peritonitis. Her chronic medications were prednisone $(2 \mathrm{mg} / \mathrm{kg})$, spironolactone, enalapril, folate, mycophenolate mofetil (MMF), oral potassium and phosphate replacement. She had received no recent blood transfusions, and her serum haemoglobin concentration was $8.1 \mathrm{~g} / \mathrm{dL}$. A non-contrast computed tomography (CT) image of her brain showed an area of hypodensity in the right cortex. Cranial magnetic resonance imaging (cMRI) revealed multiple areas of T2/ flair hyperintensity involving white matter in the posterior parietal lobes, occipital lobes and cerebellar white matter (Fig. 1). A diagnosis of PRES was made, and she was ventilated, her blood pressure was treated, and her medication and PD continued. She became more responsive, and 10 days after the onset of the initial symptoms had made an almost complete neurological recovery. She was discharged with some residual right-hand weakness, and dysarthria on maintenance sodium valproate. She died within 6 months of discharge from complications of her renal failure.

\section{Case 2}

A 9-year-old boy known to have steroid-dependent minimal-change nephrotic syndrome and hypertension was admitted with intractable seizures requiring ventilation. On admission he had elevated blood pressure $(150 / 100 \mathrm{mmHg} \text {, 50th centile } 100 / 60 \mathrm{mmHg})^{[19]}$ and normal renal function. His blood urea level was $1.5 \mathrm{mmol} / \mathrm{L}$, serum creatinine $40 \mu \mathrm{mol} / \mathrm{L}$ and serum sodium $141 \mathrm{mmol} / \mathrm{L}$, while his haemoglobin concentration was normal at $13.3 \mathrm{~g} / \mathrm{dL}$. His chronic medications were prednisone $(2 \mathrm{mg} / \mathrm{kg})$, enalapril, spironolactone, perindopril, simvastatin and MMF. A non-contrast CT image of his brain revealed cerebral oedema, and cMRI revealed T2 hyperintensity in the frontal and parietal lobes as well as watershed infarcts. He improved rapidly with management of his blood pressure. On follow-up he

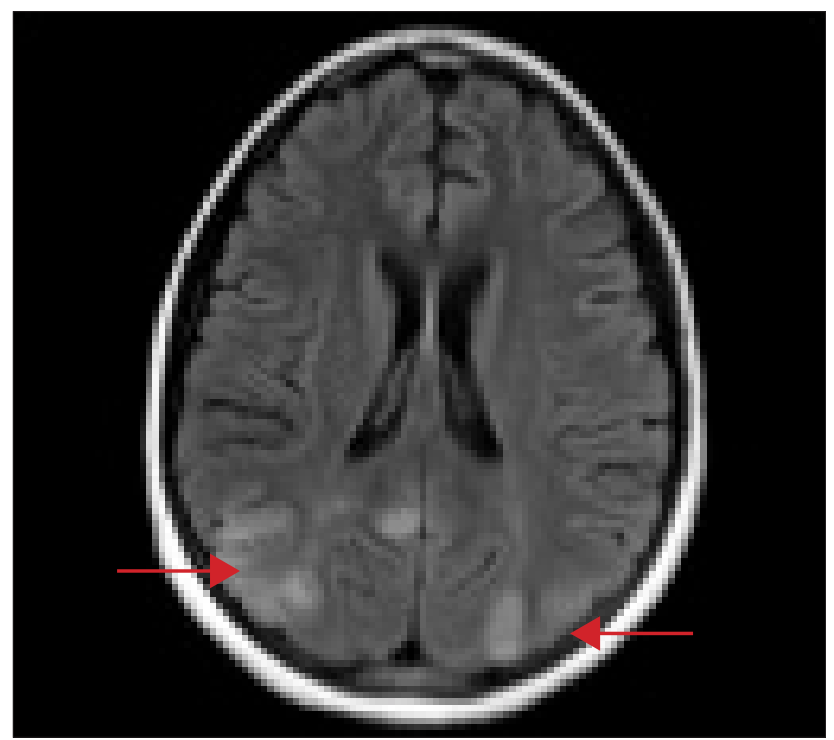

Fig. 1. Case 1. T2/flair magnetic resonance image showing multiple hyperintensities (arrows). had no residual neurological symptoms and no further seizures on maintenance sodium valproate.

\section{Case 3}

An 8-year-old girl on PD for CKD due to immune complex-mediated focal segmental glomerular sclerosis developed seizures and cortical blindness while in the ward. Her blood pressure was elevated (125/95 mmHg, 50th centile $96 / 57 \mathrm{mmHg})^{[19]}$ and she had worsening renal function with a blood urea level of $5 \mathrm{mmol} / \mathrm{L}$, serum creatinine $544 \mu \mathrm{mol} / \mathrm{L}$ and serum sodium $133 \mathrm{mmol} / \mathrm{L}$. Her haemoglobin concentration was $10.1 \mathrm{~g} / \mathrm{dL}$, and she had not received any blood transfusion. She was managed on weaning doses of prednisone (1 mg/kg), MMF, 1-alpha vitamin $\mathrm{D}$, calcium, folate, potassium, thiamine and ferrous oral supplementation, erythropoietin and omeprazole. cMRI revealed extensive white-matter disease with T2/ flair hyperintensities globally. Her symptoms resolved completely within a month of onset.

\section{Case 4}

A 10-year-old girl had newly diagnosed nephrotic syndrome with immune complex-mediated focal segmental glomerular sclerosis. Having been pulsed with methylprednisone and on prednisone ( $2 \mathrm{mg} / \mathrm{kg}$ ), she developed transient visual loss and seizures and was brought to hospital. Her blood pressure was also elevated (144/99 $\mathrm{mmHg}$, 50th centile $99 / 59 \mathrm{mmHg})^{[19]}$ and she had an elevated blood urea level of $8.6 \mathrm{mmol} / \mathrm{L}$, serum creatinine $103 \mu \mathrm{mol} / \mathrm{L}$ and serum sodium $141 \mathrm{mmol} / \mathrm{L}$. Her haemoglobin concentration was $10.6 \mathrm{~g} /$ dL. Her medication included prednisone $(2 \mathrm{mg} / \mathrm{kg})$, antituberculosis treatment, spironolactone, amlodipine, doxazosin and simvastatin. cMRI was unavailable at the time, and CT imaging showed some hypodensities in the parietal and occipital lobes. Her visual loss resolved with blood pressure control, and she is now well.

\section{Case 5}

A 10-year-old girl on PD with end-stage renal disease secondary to HIV-associated nephropathy was admitted with a history of newonset focal seizures. On admission her blood pressure was elevated $(168 / 130 \mathrm{mmHg}$, 50th centile 100/59 $\mathrm{mmHg}) .{ }^{[19]}$ Her blood urea level was $11.2 \mathrm{mmol} / \mathrm{L}$, serum creatinine $779 \mu \mathrm{mol} / \mathrm{L}$ and serum sodium $135 \mathrm{mmol} / \mathrm{L}$. Her haemoglobin concentration was $10.1 \mathrm{~g} / \mathrm{dL}$, She was on antiretroviral treatment, enalapril, amlodipine, metoprolol, folate, calcium carbonate, vitamin D, erythropoietin and prednisone $(2 \mathrm{mg} /$ $\mathrm{kg}$ ). CT imaging identified multifocal white-matter hypodensitites and a thinned cortex. cMRI T2/flair identified hyperintensities in the parietal and occipital lobes. She was started on sodium valproate, with good seizure control and no neurological deficit.

\section{Review of the literature}

We identified 12 published case reports or series describing 64 children aged between birth and 14 years of age with renal disease who had been diagnosed with PRES. ${ }^{[5-17]}$ Their mean age was 9.1 years. There were 24 males, 24 females and 16 of unreported sex. The baseline diagnoses of all the reviewed patients $(N=64)$ are shown in Fig. 2. Most reviewed patients (91\%), and all the newly reported patients, had hypertension at the time of onset of symptoms. Thirtyfive of the 64 reviewed patients were on immunosuppressive drugs, 3 were receiving haemodialysis and $5 \mathrm{PD}$, and 4 had undergone renal transplants.

Seizures were the presenting symptom in $91 \%$ of cases, an altered level of consciousness in $63 \%$, headaches in $50 \%$ and visual disturbances in $41 \%$ (Fig. 3). The radiological cMRI diagnosis mostly involved changes 


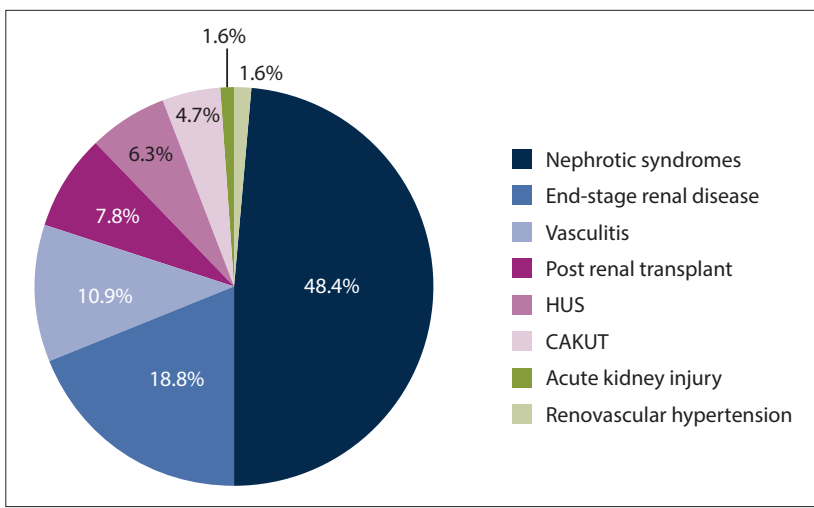

Fig. 2. Baseline diagnoses of paediatric patients with renal posterior reversible encephalopathy syndrome $(\mathrm{N}=64)$. (HUS = haemolytic uraemic syndrome; $C A K U T=$ congenital abnormalities of the kidney and urinary tract.)

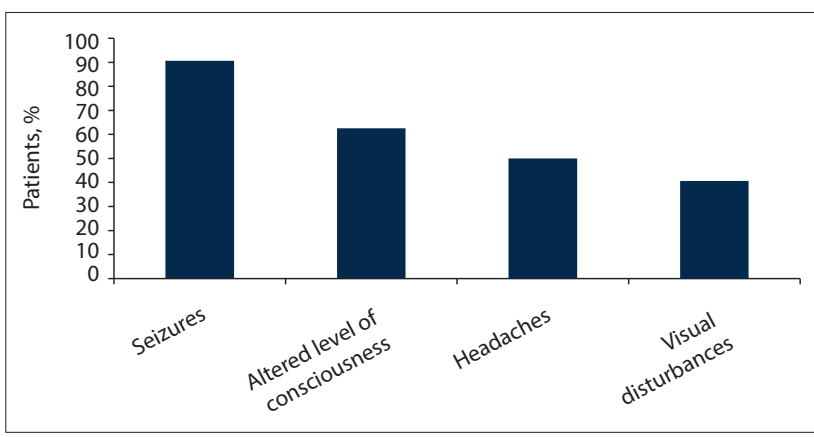

Fig. 3. Presenting symptoms of paediatric patients with renal posterior reversible encephalopathy syndrome $(\mathrm{N}=64)$.

in the occipital lobe (91\%), but also in the parietal lobe (73\%), frontal lobe (36\%) and temporal lobe (39\%) (Fig. 4). Complete recovery was reported in $87.5 \%$ of cases, residual epilepsy in $5 \%$ and recurrence of PRES in 5\%; there was one report of other non-specified neurological sequelae, and one patient was lost to follow-up.

\section{Discussion}

The five newly reported cases are similar to those reviewed in the literature in terms of age at presentation (mean 9.6 years, literature review 9.5 years). There were equal numbers of female and male patients in the 64 reviewed children, but four of the five newly reported cases were female. Because of the limited cohort size it is difficult to comment on the significance of this finding, but it is in keeping with existing literature reporting that more females than males develop PRES. ${ }^{[5]}$

Four of the five reported cases had nephrotic syndrome-related diagnoses, while $48 \%$ of the reviewed cases were similarly afflicted (although this figure may be higher, as 19\% of the reviewed cases were reported to be in end-stage renal disease without a specified underlying diagnosis). Three of the five reported cases were in endstage renal disease and undergoing renal replacement therapy, and only one patient had a normal estimated glomerular filtration rate. This represents a higher proportion of patients with advanced renal disease than that reflected in the literature. The development of PRES as a complication of PD or uraemic encephalopathy therefore does not fully explain the pathogenesis in all the cases.

Only one of the five reported cases and $11 \%$ of the reviewed cases had been diagnosed with systemic vasculitis at the time of PRES diagnosis. In keeping with the hypothesis that the pathogenesis of PRES is related to endothelial inflammation, ${ }^{[4]}$ this would suggest that

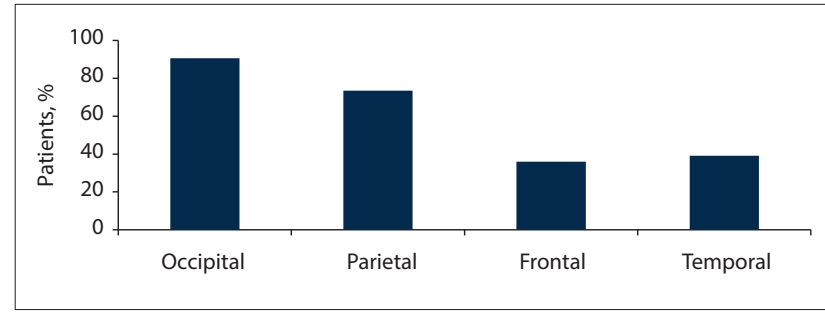

Fig. 4. Patterns of magnetic resonance imaging changes at diagnosis of posterior reversible encephalopathy syndrome $(\mathrm{N}=64)$.

a process other than vasculitis caused the implicated inflammation in these cases.

Importantly, all the reported patients and $91 \%$ of the reviewed patients were hypertensive at the time of PRES diagnosis. Only one patient diagnosed with renovascular hypertension was diagnosed with PRES in the reviewed literature. All the reported patients were hypertensive on medication prior to symptom development. However, it is surprising that no patient with Takayasu's arteritis or other renovascular hypertensive disease seen in our department has developed PRES. We postulate that PRES may be related to the rate of rise of blood pressure instead of the sustained levels of hypertension in these patients.

Although PRES associated with blood transfusion has been reported in the literature, ${ }^{[20]}$ this was not observed in our patients. One of the five reported patients had a haemoglobin concentration $<10 \mathrm{~g} / \mathrm{dL}$, and none had received blood transfusion prior to the development of symptoms. This was not universally reported on in the reviewed patients. All the reported patients were on prednisone at the time of onset of symptoms, and two were on other immunosuppressive therapy (MMF). All the reported patients had normal serum sodium levels.

The most common presenting symptom in the literature was seizures (91\%), and all the reported patients presented with seizures. There are reports that PRES may be considered an epileptic disorder, ${ }^{[18]}$ and that it could be monitored or predicted by electroencephalography, which would show either focal non-convulsive seizures or status epilepticus. However, three of the five reported patients were not treated with maintenance antiepileptic drugs and have not had recurrences of seizures. The need for maintenance antiepileptic medication should be reviewed, especially in this patient group, as they require multiple drugs for renal failure and the underlying disease.

The pattern of cMRI changes at PRES diagnosis reviewed in the literature showed a predominance of occipital and parietal changes, which is consistent with the reported cases in which this imaging modality was available. It also to some extent explains the frequency of visual disturbances present at the time of diagnoses (three of six of the reported cases, and $41 \%$ of the reviewed cases). Most (87.5\%) of the reviewed cases had radiological recovery as seen on follow-up MRI. Repeat imaging was not requested in the reported patients

The follow-up of our five patients ranged from a few months to many years, with a mean of 28 months. All five of these patients recovered from their presentation with PRES, four without any neurological deficit. There were no reported recurrent episodes. The literature review revealed complete recovery in $87.5 \%$ of patients. This good recovery suggests that PRES is a transient syndrome with a favourable outcome if identified correctly and managed appropriately.

\section{Study limitations}

This was a retrospective analysis of medical records spanning a 17 -year period. Selection bias is likely to have been introduced because only patients referred to the renal service were included. 


\section{Recommendations}

Based on our experience and a review of the literature:

- The good prognosis associated with PRES should be considered when a decision about intensive care in low-resource settings is made.

- Repeat MRI is probably not indicated in patients who have undergone a full neurological recovery.

- The need for maintenance antiepileptic medication should be determined.

- The usefulness and availability of electroencephalography should be determined in this population.

\section{Conclusions}

All the reported cases of PRES presented clinically as hypertensive emergencies and radiologically with PRES, and most had a complete neurological recovery. These case reports support the hypothesis that PRES represents the radiological manifestation of a hypertensive emergency, now identified since the advent of MRI technology. However, the question why this syndrome does not seem to occur in patients with Takayasu's arteritis and other renovascular hypertensive disease, where hypertension is frequently severe, highlights the probability that there are as yet unidentified processes that result in the condition. The need for maintenance antiepileptic treatment remains to be determined.

Acknowledgements. The patients and parents at Chris Hani Baragwanath Hospital.

Author contributions. JKS: Concept of study; JKS, KLP, UK: Data collection, data analysis, final manuscript approval.

Funding. None.

Conflicts of interest. None.

1. Hinchey J, Chaves C, Appignani B, et al. A reversible posterior leukoencephalopathy syndrome. N Engl J Med 1996;334(8):494-500. https://doi.org/10.1056\%2Fnejm199602223340803
2. Fugate JE, Rabinstein AA. Posterior reversible encephalopathy syndrome: Clinical and radiological . Fugate JE, Rabinstein AA. Posterior reversible encephalopathy syndrome: Clinical and radiological
manifestations, pathophysiology, and outstanding questions. Lancet Neurol 2015;14(9):914-925. https:// doi.org/10.1016\%2Fs1474-4422\%2815\%2900111-8

3. Yamamoto H, Natsume J, Kidokoro H, et al. Clinical and neuroimaging findings in children with posterior reversible encephalopathy syndrome. Eur J Paediatr Neurol 2015;19(6):672-678. https://doi. org/10.1016\%2Fj.ejpn.2015.07.005

4. Fischer M, Schmutzhard E, Posterior reversible encephalopathy syndrome. J Neurol 2017;264(8):16081616. https://doi.org/10.1007/s00415-016-8377-8

5. Faille LD, Fieuws S, van Paesschen W. Clinical predictors and differential diagnosis of posterior reversible encephalopathy syndrome. Acta Neurol Belg 2017;117(2):469-475.

6. Akin F, Kilicaslan C, Solak ES, Uzun M, Aygun S, Arslan S. Posterior reversible encephalopathy syndrome in children: Report of three cases. Childs Nerv Syst 2014;30(3):535-540. https://doi.org/10.1007\%2Fs00381in children:

7. Chen TH, Lin WC, Tseng YH, Tseng CM, Chang TT, Lin TJ. Posterior reversible encephalopathy syndrome in children: Case series and systematic review. J Child Neurol 2013;28(11):1378-1386. https:// syndrome in children: Case series and systent

8. El-Naggari MA, Al-Nabhani D, El-Nour I, El-Manzalawy A, Abdelmogheth AA. Posterior reversible El-Naggari MA, Al-Nabhani D, El-Nour I, El-Manzalawy A, Abdelmogheth AA. Posterior reversible
encephalopathy syndrome in two Omani children with underlying renal diseases. Sultan Qaboos Univ Med J 2015;15(3):e424-e428. https://doi.org/10.18295\%2Fsqumj.2015.15.03.020

9. Gumus H, Per H, Kumandas S, Yikilmaz A. Reversible posterior leukoencephalopathy syndrome in 9. Gumus H, Per H, Kumandas S, Yik imaz A. Reversible posterior leukoencephalopathy syndrome in
childhood: Report of nine cases and review of the literature. Neurol Sci 2010;31(2):125-131. https://doi. org/10.1007\%2Fs10072-009-0158-Z

10. Gera DN, Patil SB, Iyer A, et al. Posterior reversible encephalopathy syndrome in children with kidney . Gera DN, Patil SB, lyer A, et al. Posterior reversible encephalopathy syndrome in child
disease. Indian J Nephrol 2014;24(1):28-34. https://doi.org/10.4103\%2F0971-4065.125053

1. Ito I, Nishida M, Morioka S, et al. A case of steroid-resistant nephrotic syndrome associated with systemic 1. Ito I, Nishida M, Morioka S, et al. A case of steroid-resistant nephrotic syndrome associated with
lupus erythematosus. Lupus 2011;23(7):703-706. https://doi.org/10.1177\%2F0961203311432740

12. Ishikura K, Ikeda M, Hamasaki Y, et al. Posterior reversible encephalopathy syndrome in children: Its Ishikura K, Ikeda M, Hamasaki Y, et al. Posterior reversible encephalopathy syndrome in children: Its
high prevalence and more extensive imaging findings. Am J Kidney Dis 2006;48(2):231-238. https://doi. high prevalence and more extensive

13. Incecik F, Herguner MO, Altunbasak S, Erbey F, Leblebisatan G. Evaluation of nine children with Incecik F, Herguner MO, Altunbasak S, Erbey F, Leblebisatan G. Evaluation of nine children with
reversible posterior encephalopathy syndrome. Neurol India 2009;57(4):475-478. https://doi.
org $/ 10.4103 \% 2 \mathrm{~F} 0028-3886.55605$

14. Ohta T, Sakano T, Shiotsu M, et al. Reversible posterior leukoencephalopathy in a patient with Wegener granulomatosis. Pediatr Nephrol 2004;19(4):442-444. https://doi.org/10.1007\%2Fs00467-003-1286-y

15. Onder AM, Lopez R, Teomete U, et al. Posterior reversible encephalopathy syndrome in the pediatric renal Onder AM, Lopez R, Teomete U, et al. Posterior reversible encephalopathy syndrome in the pediatric
population. Pediatr Nephrol 2007;22(11):1921-1929. https://doi.org/10.1007\%2Fs00467-007-0578-z

population. Pediatr Nephrol 2007;22(11):1921-1929. https://doi.org/10.1007\%2Fs00467-007-0578-z
16. Endo A, Fuchigami T, Hasegawa M, et al. Posterior reversible encephalopathy syndrome in childhood: R. Endo A, Fuchigami T, Hasegawa M, et al. Posterior reversible encephalopathy syndrome in childhood:
Report of four cases and review of the literature. Pediatr Emerg Care 2012;28(2):153-157. https://doi.org/1 Report of four cases and review of
$0.1097 \% 2 \mathrm{Fpec} .0 \mathrm{~b} 013 \mathrm{e} 3182442 \mathrm{fe} 8$

17. Yamada A, Atsumi M, Tashiro A, Hiraiwa T, Ueda N. Recurrent posterior reversible encephalopathy

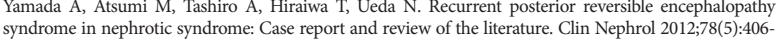
syndrome in nephrotic syndrome: Case

18. Grioni D, Pavan F, Prunotto G, Canonico F, Grandi C, Rovelli A. Should posterior reversible encephalopathy syndrome be mainly considered an epileptic disorder? Results of a sequential neurophysiological study in a syndrome be mainly considered an epileptic disorder? Results of a sequential neurophysiological
pediatric cohort. Neuropediatrics 2017;48(2):72-78. https://doi.org/10.1055\%2Fs-0037-1598251

19. Blood pressure levels for boys and girls by age and height percentile. https://www.nhlbi.nih.gov/files/docs/ guidelines/child_tbl.pdf (accessed 28 April 2017).

20. Raj S, Overby P, Erdfarb A, Ushay HM. Posterior reversible encephalopathy syndrome: Incidence and Raj S, Overby P, Erdfarb A, Ushay HM. Posterior reversible encephalopathy syndrome: Incidence and
associated factors in a pediatric critical care population. Pediatr Neurol 2013;49(5):335-339. https://doi. org/10.1016\%2Fj.pediatrneurol.2013.06.007

Accepted 26 September 2017 